\title{
Naturheilkundlich-integratives Behandlungskonzept bei Colitis ulcerosa. Eine Kasuistik
}

Sarah Gabriel, Jost Langhorst

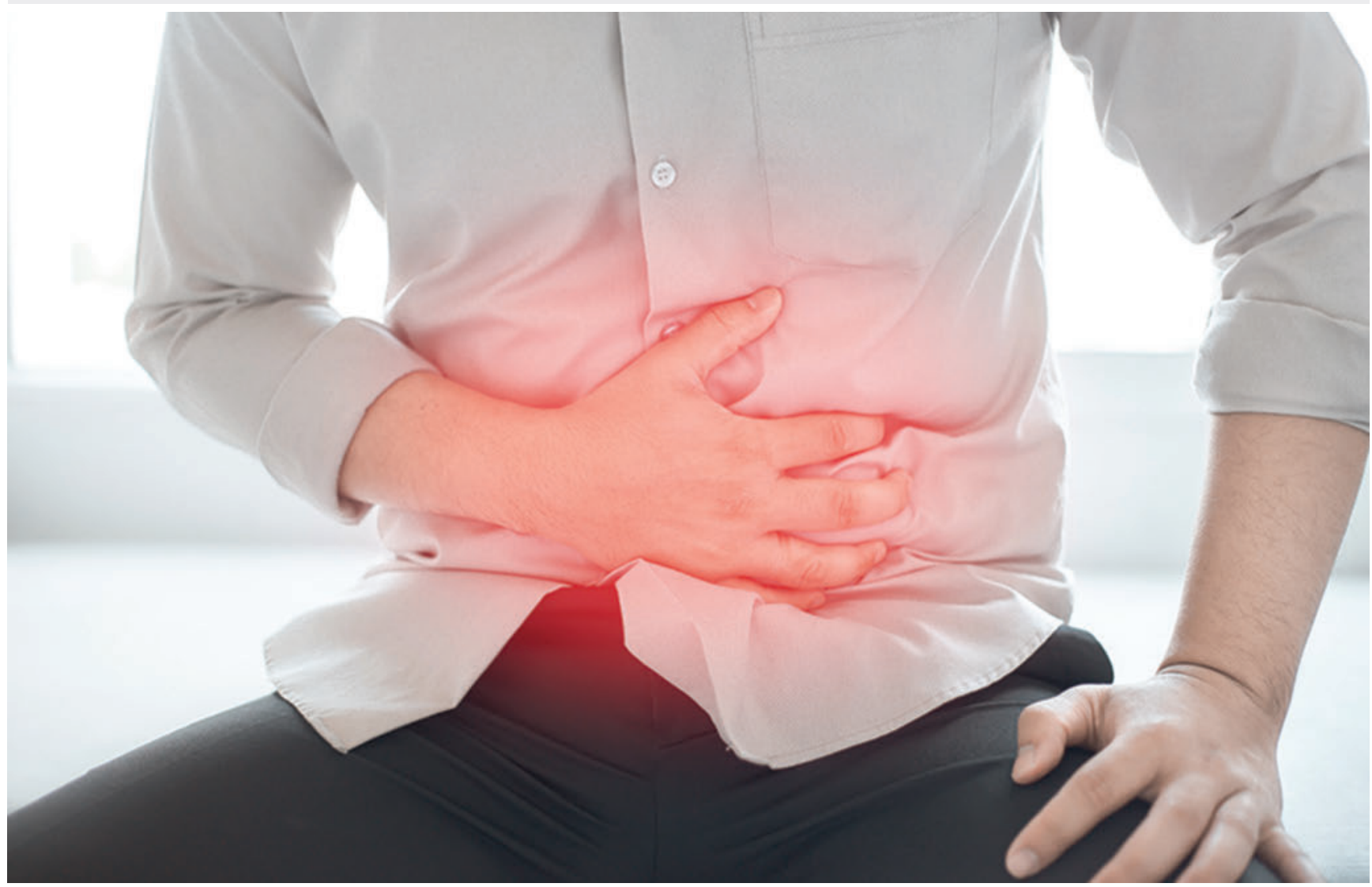

Als ergänzende Maßnahmen kommen bei Colitis ulcerosa gemäß Leitlinie Phytotherapie, Mind-Body-Verfahren zur Stressreduktion sowie Akupunktur im leichten bis moderaten Schub infrage. Foto: Adobe Stock / chompoo

In Deutschland sind etwa 150000 Personen an einer Colitis ulcerosa erkrankt. Trotz des bekannten Krankheitsbildes erhalten sie nicht immer eine adäquate Therapie. Die folgende Falldarstellung beschreibt eine integrative Behandlung im stationären Setting.

\section{Anamnese}

Ein 64-jähriger Patient wurde im akuten Schub einer Colitis ulcerosa mit täglich 15 wässrig-breiigen, zum Teil blutigen Diarrhoen zur Diagnostik und zur stationären multimodalen naturheilkundlich-integrativen Therapie aufgenommen. Die Erstdiagnose der Colitis ulcerosa erfolgte 1991. Bis 2014 zeigte sich eine nur geringe Krankheitsaktivität. Seit 2014 kam es zu einer deutlichen Zunahme der Krankheitsaktivität, ohne dass dem Patienten hierfür ein Grund ersichtlich war. Der aktuelle Schub besteht seit 3 Monaten und entspricht endoskopisch dem Bild einer akuten Linksseitenkolitis mit mittelgradiger Aktivität entsprechend der Klassifikation mittels
Mayo-Subscore Endoskopie. In der Histologie imponierte eine akute Entzündung entsprechend Grad 3 im Nancy Index. Das Calprotectin im Stuhl betrug zu diesem Zeitpunkt $574 \mu \mathrm{g} / \mathrm{g}($ Referenzwert $<50 \mu \mathrm{g} / \mathrm{g})$. Bei fehlendem Ansprechen auf die Basismedikation in Form eines topischen und eines systemischen 5-Aminosalizylats wurde eine Therapie mit dem Biologicum Adalimumab etabliert. Bisher jedoch ohne nennenswerten Einfluss auf die Symptomatik.

Bekannt sind ein primärer arterieller Hypertonus, eine Hypercholesterinämie und ein Diabetes mellitus Typ 2, Erstdiagnose 2014, dessen Therapie mit Glibenclamid im 
>Tab. 1 Medikation bei stationärer Aufnahme.

\begin{tabular}{|c|c|c|c|c|c|}
\hline Medikamentenname & Applik. & morgens & mittags & abends & nachts \\
\hline Metformin $1000 \mathrm{mg}$ & p.o. & 1 & 0 & 1 & 0 \\
\hline Sitagliptin $100 \mathrm{mg}$ & p.o. & 0 & 0 & 1 & 0 \\
\hline Ramipril $5 \mathrm{mg}$ & p.o. & $1 / 2$ & 0 & 0 & 0 \\
\hline Atorvastatin $20 \mathrm{mg}$ & p.o. & 0 & 0 & 1 & 0 \\
\hline $\begin{array}{l}\text { Mesalazin (Salofalk Suppositorien / } \\
\text { Schaum } 1 \mathrm{~g} \text { ) }\end{array}$ & rektal & 0 & 0 & 0 & 1 \\
\hline Adalimumab 40 mg, alle 2 Wo. & s.c. & 0 & 1 & 0 & 0 \\
\hline
\end{tabular}

Dezember 2018 bei einem $\mathrm{HbA}_{1 \mathrm{c}}$ von > 8\% auf Sitagliptin / Metformin umgestellt wurde. Bereits bei einem Blutzuckerwert von $95 \mathrm{mg} / \mathrm{dl}$ beschreibt der Patient Symptome einer Hypoglykämie mit Kaltschweißigkeit, Tremor und Unruhe. In den letzten 1,5 Jahren kam es zu einem ungewollten Gewichtsverlust von $18 \mathrm{~kg}$. Für eine maligne Grunderkrankung fand sich im Rahmen der ambulanten Vordiagnostik kein Hinweis.

Der Patient ist verheiratet, in einem sozialen Beruf in Teilzeit tätig und beschreibt ein hohes Maß an Bewegung im Alltag, jedoch ohne gezielte Trainingsformen. Bezüglich Ernährung und Entspannungsverfahren ist der Patient insgesamt eher unerfahren. Es werden keine schwerwiegenden kritischen Lebensereignisse angegeben. Im Vordergrund stehen bei Aufnahme besonders die Entwicklung von Bewusstheit für Zusammenhänge von Lebensstil und Symptomatik sowie eine Ressourcenaktivierung durch Entspannung und Achtsamkeit.

\section{Klinischer Untersuchungsbefund}

64-jähriger Patient in reduziertem Allgemeinzustand und schlankem Ernährungszustand, wach, orientiert, adäquat schwingungsfähig.

- Größe: 1,76 m; Gewicht: 61 kg; BMl: 19,7 kg/m²; Allergie: keine bekannt; Konstitution: leptosom.

- Kopf/Hals: Hirnnerven inkl. POM unauffällig, Tonsillen klein, Rachen reizlos, Zunge bland, Carotiden frei, Schilddrüse nicht vergrößert tastbar.

- Cor: normofrequente, rhythmische Herzaktion, keine vitientypischen Geräusche.

- Pulmo: Vesikularatmung, sonorer Klopfschall, Lungengrenzen normal.

- Abdomen: Lebhafte Darmgeräusche, weiche Bauchdecke, kein Druckschmerz, keine Abwehrspannung, keine Resistenzen, Leber und Milz nicht vergrößert tastbar.

- Extremitäten: frei und umfänglich beweglich, periphere Pulse gut tastbar, grob neurologisch (PSR und BSR prompt auslösbar) unauffällig.

\section{Laborbefund bei stationärer Aufnahme}

$\mathrm{HbA}_{1 \mathrm{c}}$ 6,9\% (Normbereich<5,7\%), Glucose $159 \mathrm{mg} / \mathrm{dl}$ $(60-100 \mathrm{mg} / \mathrm{dl})$, CRP 0,79 mg/dl $(<0,5 \mathrm{mg} / \mathrm{dl})$; alle weiteren im Rahmen eines internistischen Aufnahmestatus erhobenen Parameter befanden sich im Normbereich. Medikation s. - Tab. 1.

\section{Weiterführende Diagnostik}

\section{Stuhldiagnostik}

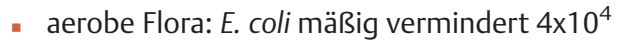

- anaerobe Flora: Clostridium sp. mäßig vermehrt 1×10

- Pilze: im Normbereich

- Stuhl-pH: sauer bei 5,5

\section{Verdauungsparameter}

- Pankreaselastase 188 (> $200 \mu \mathrm{g} / \mathrm{g})$

\section{Verdauungsrückstände}

- vermehrt Neutralfette, keine Fettsäuren als Hinweis für exokrine Pankreasinsuffizienz

\section{Schleimhautmarker}

- Calprotectin $564(<50 \mu \mathrm{g} / \mathrm{g})$

- Lactoferrin > $100(<7,25 \mu \mathrm{g} / \mathrm{g})$

Ruhe-EKG: unauffällig

\section{Therapie}

Der Patient wurde während seines 14-tägigen stationären Aufenthalts in ein komplexes multimodales naturheilkundlich-integratives Therapieregime eingebunden. Ziel war die Verminderung der abdominellen Symptomatik und die Verbesserung der metabolischen Situation. Das Therapiekonzept beinhaltete neben intensivierter Anleitung zu Entspannungsverfahren, Ernährungs- und Bewegungstherapie ebenso hydrotherapeutische Verfahren, Phytotherapie, Akupunktur und segmentale Bindegewebs- und Faszientherapie (Schröpfkopfmassagen). 
Als medikamentöse Therapie erhielt der Patient ergänzend zu seiner vorbestehenden Medikation ( $\triangleright$ Tab. 1) ein Kombinationspräparat aus Myrrhe, Kamillenblütenextrakt und Kaffeekohle zur Therapie der akuten Diarrhoe im Rahmen der Colitis ulcerosa. Die Dosierung betrug $3 \times 4$ Tabletten / Tag. Des Weiteren täglich $2 \times 1$ Teelöffel indische Flohsamenschalen als Quellstoff zur Darmregulation. Abends wurde zusätzlich ein Naturheilkunde-Klysma angewärmt verabreicht, als $10 \%$ iger Dekokt bestehend aus 37,5g Weidenrinde, Eichenrinde und Tormentillwurzel, 61g Aqua, $0,2 \mathrm{~g}$ Kaliumsorbat sowie $1,5 \mathrm{~g}$ Johannisbrotkernmehl. Ergänzt wurde die Medikation bei Mangel an E. coli Nissle um Mutaflor, welches zur probiotischen Therapie und zur Remissionsförderung bei Colitis ulcerosa einschleichend mit dem Ziel der Dosierung zweimal täglich verabreicht wurde. Hierunter sistierten die blutigen Diarrhoen bei nur tendenziell verringerter Anzahl der Stühle.

Bei im Verlauf zunächst nur tendenziell gebesserten Diarrhoen zeigten sich bei verminderter Pankreaselastase Hinweise auf eine exokrine Pankreasinsuffizienz in der Stuhldiagnostik. Die Bildgebung in Form einer Oberbauchsonografie zeigte keine strukturellen Auffälligkeiten des Pankreas. Vor diesem Hintergrund wurde die Therapie ernährungsadaptiert um 2 Kaps. Rizoenzyme ausgeweitet. Darunter verbesserten sich Stuhlkonsistenz und -frequenz rasch.

Sehr profitiert hat der Patient von der Umstellung der Kost auf eine pflanzenbasierte, mediterrane Vollwertkost. Hierunter kam es zu einer Stabilisierung der metabolischen Situation mit deutlicher Reduktion der Blutzuckerwerte, sodass die Metformindosis von $2000 \mathrm{mg}$ auf $1000 \mathrm{mg}$ halbiert werden und Atorvastatin bei normwertigen Blutfettwerten abgesetzt werden konnte. Auch die Blutdruckwerte normalisierten sich im Verlauf, sodass Ramipril ebenfalls abgesetzt werden konnte.

Der Patient profitierte vom ganzheitlichen Ansatz der Behandlung, konnte Zusammenhänge der psychischen und somatischen Symptome sowie deren gegenseitige Beeinflussung besser erkennen und erlangte Fähigkeiten im Umgang damit. Unter dieser naturheilkundlichen Komplextherapie mit Schwerpunkt auf Behandlung der Colitis waren die Beschwerden um etwa $70 \%$ rückläufig.

Insgesamt profitierte der Patient vom stationären Aufenthalt mit einer Normalisierung der Verdauungsaktivität, einer Reduktion der Stuhlfrequenz auf 2-3 breiig bis geformte Stühle und einer deutlichen Verbesserung der metabolischen Situation. Es wurde die Empfehlung ausgesprochen, die Adalimumab-Therapie im Rahmen des Integrativen Versorgungsansatzes weiterzuführen, da der therapeutische Effekt erst mit einer Latenz von 3 Monaten voll ausgebildet und zu erwarten ist.

\section{Diskussion}

Patienten mit Colitis ulcerosa leiden im akuten Schub häufig unter imperativem Stuhldrang mit hoher Stuhlfrequenz und Blutabgängen. Dadurch sind das Allgemeinbefinden und die Lebensqualität oft massiv eingeschränkt. Im Gesamtkontext der Fallbetrachtung ist zu betonen, dass der Patient ein zweiwöchiges multimodales naturheilkundlich-integratives Behandlungskonzept mit den oben beschriebenen Modulen enthielt. Im Rahmen der Diskussion werden die Therapiesäulen Ernährung und die Phytotherapie weiter im Detail beleuchtet.

Als eine Therapiesäule zur unmittelbaren Symptomreduktion und -kontrolle ergänzend zur konventionellen medikamentösen Therapie wurden bei diesem Patienten ergänzend zu Lebensstilfaktoren verschiedene Phytotherapeutika in die Therapie integriert. Zum Einsatz kamen fein vermahlene Flohsamenschalen. Sie wirken als Quellmittel (Quellzahl 40) und generieren eine leichte antientzündliche Wirkung über die Fermentierung von kurzkettigen Fettsäuren. Zum Remissionserhalt werden 1-3 Gaben von jeweils $5 \mathrm{~g}$ täglich empfohlen. Für den Patienten bestand die optimale Dosis in $2 \times 5 \mathrm{~g}$ täglich.

Unterstützend erhielt der Patient ein Kombinationsarzneimittel aus Myrrheharz $(100 \mathrm{mg})$, Kaffeekohle $(50 \mathrm{mg})$ und Kamillenblütenextrakt $(70 \mathrm{mg})$, welches antiphlogistisch, antidiarrhoisch und antimikrobiell wirkt und für das nach aktueller Studienlage Hinweise dafür vorliegen, dass es in der remissionserhaltenden Therapie einer Standardtherapie mit Mesalazin nicht unterlegen ist. Die Standarddosierung ist $3 \times 4$ Dragees/Tag. Sowohl Flohsamen als auch die Kombination aus Myrrheharz, Kamillenextrakt und Kaffeekohle werden im Kapitel Komplementärmedizin der aktuellen AWMF S3-Leitlinie für Colitis ulcerosa genannt.

Ergänzend hat sich bei Colitis ulcerosa im klinischen Alltag der Einsatz eines Dekokts aus Tormentillwurzel, Weidenrinde und Eichenrinde in Form eines Klysmas bewährt, der abends (angewärmt) verabreicht über die Nacht auf die Darmschleimhaut einwirken kann. Darunter kann eine adstringierende, antioxidative und antiseptische Wirkung erreicht werden. Die Weidenrinde enthält u. a. Salicin, ähnlich der Acetylsalicylsäure. Salicin wird durch die Darmflora in Salicylsäure umgewandelt und hat entzündungshemmende Eigenschaften. Eichenrinde enthält neben entzündungshemmenden Flavonoiden v.a. Catechine und Tannine - Gerbstoffe, die in Wechselwirkung mit Eiweißmolekülen treten und somit einen adstringierenden Effekt auf Schleimhäute und Blutgefäße haben. Dadurch können wie im hier vorliegenden Fall die Wundheilung beschleunigt und Blutungen eher gestillt werden. Auch die Tormentillwurzel enthält Gerbstoffe und entzündungshemmende sekundäre Pflanzenstoffe, die ebenfalls bei Schleimhautläsionen und -reizungen lindernd wirken. 
Insgesamt ist der Einsatz pflanzlicher Präparate in Deutschland auch in der Therapie chronisch entzündlicher Darmerkrankungen verbreitet. In Studien berichtet bis zu jeder vierte Patient in Deutschland von der Nutzung phytotherapeutischer Therapieoptionen, auch wenn bisher nur für einige Medikamente klinische Studien mit Indikation CED vorliegen. Nachdem unter dieser Therapie die Blutungen im Darm sistierten, wurde durch den Nachweis einer verminderten Pankreaselastase die Diagnose einer exokrinen Pankreasinsuffizienz gestellt, ohne dass in der Bildgebung strukturelle Auffälligkeiten des Pankreas nachzuweisen waren. Unter der Therapie mit Rizoenzymen (Verdauungsenzymen der Bauchspeicheldrüse aus Reispilzkulturen) verbesserten sich Stuhlkonsistenz und -frequenz weiter. Die exokrine Pankreasinsuffizienz ist bei chronischen Diarrhoen eine wichtige Differentialdiagnose, bei der von einer relevanten Dunkelziffer auszugehen ist. Im hier geschilderten Fall gelang es, unter der ernährungsadaptierten Therapie einen weiteren wichtigen therapeutischen Impuls zu geben.

Als ein weiterer zentraler Punkt für die Therapie wird der Einfluss der Ernährung bei CED zunehmend anerkannt. Die Bedeutung für das Metabolische Syndrom ist ohnehin kaum zu überschätzen. Die Ernährung ist vor allem in der jüngeren Vergangenheit durch den Aufschwung der Mikrobiom-Forschung wieder Gegenstand der konventionellen wissenschaftlichen Betrachtungen geworden. Ziel ist es u.a. durch diätetische, antientzündliche Maßnahmen den Krankheitsverlauf positiv zu beeinflussen. Der Patient erlernte im stationären Alltag die Umsetzung einer pflanzenbasierten, mediterranen Vollwerternährung, die auf die speziellen Bedürfnisse bei CED zugeschnitten ist. Gemieden werden sehr fettige, scharfe, süße, saure oder salzige Speisen und die Zufuhr an tierischem Protein wird reduziert. Stattdessen werden regionale und saisonale Bioprodukte verwendet, die möglichst wenig verarbeitet und schonend zubereitet werden. Dadurch kann ein hohes Maß an Phytaminen und antientzündlichen, antioxidativen Wirkstoffen gewährleistet werden.

Beispielsweise enthalten Beeren- und Zitrusfrüchte Flavonoide, die eine intakte Barrierefunktion der Darmschleimhaut unterstützen. Ausreichend Ballaststoffe versorgen die Epithelzellen mit Butyraten; diese kurzkettigen Fettsäuren dienen als Energiequelle zur Ernährung der Darmschleimhaut und als protektiver Faktor gegen eine maligne Entartung. Unter anderem wird 
pathogenen Keimen das Durchdringen der Darmbarriere erschwert, sodass der Entzündungsprozess abklingen und eine Remission unterstützt werden kann.

Verstärkt wird dieser Prozess durch das Meiden von entzündungsfördernden Nahrungsmitteln, wie Proteinen tierischen Ursprungs (Fleisch- und Wurstwaren) oder Weizen (Amylase-Trypsin-Inhibitoren). Stattdessen erhalten die Patienten hochwertige Pflanzenöle, die reich an Omega3-Fettsäuren sind und sie werden bei CED zusätzlich durch Omega-3-Präparate unterstützt, die einen antientzündlichen Effekt generieren. Weizen ist bekannt für seine immunmodulierende Wirkung, z.B. aufgrund der enthaltenen Amylase-Trypsin-Inhibitoren, sodass der Patient geschult wird, auf andere Vollkornprodukte zurückzugreifen.

Gleichzeitig bewirkte die Ernährungsumstellung bei dem hier vorgestellten Patienten auch einen positiven Einfluss auf die metabolische Situation mit Reduktion von Blutdruck, Blutfettwerten und des Blutzuckers. Im hier geschilderten Fall konnte u. a. die orale Antidiabetikadosis halbiert werden. Als bekannteste Nebenwirkung bei Metformin gelten gastrointestinale Beschwerden, wie Durchfälle, sodass eine Reduktion oder wenn möglich das Absetzen ebenfalls einen positiven Einfluss auf die Stuhlfrequenz und -konsistenz haben kann. Diese Nebenwirkung ist ebenso bei Statinen bekannt, sodass der Einsatz abhängig von der Beurteilung des kardiovaskulären Risikoprofils kritisch zu hinterfragen und in jedem Fall eine Lebensstilmodifikation anzustreben ist.
Interessenkonflikt

Forschungsunterstützung: Steigerwald Arzneimittelwerke GmbH, Falk Foundation, TechLab, Inc., Dr. Willmar Schwabe, Repha GmbH biologische Arzneimittel. Vortragshonorar: Falk Foundation; MSD Sharp\&Dohme GmbH; Repha GmbH biologische Arzneimittel; Celgene GmbH; Dr. Willmar Schwabe; AbbVie. Berater/Gutachtertätigkeit: Steigerwald Arzneimittelwerke $\mathrm{GmbH}$; Repha GmbH; Ferring Arzneimittel GmbH; Sanofi. Forschungsunterstützung Stiftungen: Melinda- und Bill Gates Foundation, Karl- und Veronica Carstens-Stiftung, Ruth- und Klaus-Bahlsen-Stiftung, Dr. Heinz Horst Deichmann Stiftung, Raßfeld-Stiftung

\section{Korrespondenzadresse}

Prof. Dr. med. Jost Langhorst

Klinik für Integrative Medizin und Naturheilkunde Sozialstiftung Bamberg, Klinikum am Bruderwald Buger Straße 80

96049 Bamberg

E-Mail: jost.langhorst@sozialstiftung-bamberg.de

Literatur

Literatur bei den Autoren.

Bibliografie

DOI https://doi.org/10.1055/a-1015-5107

Zeitschrift für Phytotherapie 2019; 40: 265-269

(c) Georg Thieme Verlag KG Stuttgart · New York ISSN 0722-348X 